\title{
ORBIS: A Reconfigurable Hybrid Optical Metropolitan Area Network Architecture
}

\author{
Yufeng Xin ${ }^{1}$, Ilia Baldine ${ }^{1}$, Mark Cassada ${ }^{1}$, Daniel Stevenson ${ }^{1}$, \\ Laura E. Jackson ${ }^{2}$, and Harry Perros ${ }^{2}$ \\ 1 ANR, MCNC-RDI, RTP NC 27709 USA \\ ${ }^{2}$ Computer Science, NC State University, Raleigh NC 27609 USA
}

\begin{abstract}
In this paper, we propose a novel metropolitan area network (MAN) ring architecture, ORBIS, a multiple-service WDM platform that supports multiple transport techniques. The WDM ring is horizontally divided into multiple subnetworks, each of which supports one type of the traffic with a subset of the total wavelengths. The system and subnetwork control mechanism and reconfiguration issues are addressed in this paper.
\end{abstract}

\section{Introduction}

The challenges faced by metropolitan WDM networks are more complicated than those in the WDM backbones due to the existence of traffic from multiple transport protocols with variable bandwidth and QoS requirements [4].

Despite of the multitude of traffic, previous and current solutions are via a layered architecture on the top of a single transport platform such as SONET/SDH. This is a very expensive and not scalable approach because of the overhead of multiple layers and the complicated adaptation and encapsulation processes.

The WDM technique has provided another dimension to provide multiple services in an integrated network. By taking the advantage of the multiple wavelengths existing in a single link, we propose a novel hybrid MAN architecture, ORBIS, in which, multiple transport networks co-exist in a single WDM ring and each subnetwork is provisioned with a part of the total wavelengths. The merit behind this architecture is that different types of user traffic with different QoS and cost requirements can be simply dispatched to different optical transport subnetworks with different QoS guarantees and cost functions.

This is also a highly scalable network architecture. A piece of multitude traffic would first be admitted to an adequate subnetwork in a coarse QoS scale. Finer bandwidth and QoS provisioning can be provided inside each subnetwork. Dynamic network reconfiguration could be done via a simple way like re-allocating wavelengths to subnetworks. Finally, it allows a graceful deployment of new technologies in the same fiber infrastructure while protecting the investment on the old technologies like SONET/SDH. 


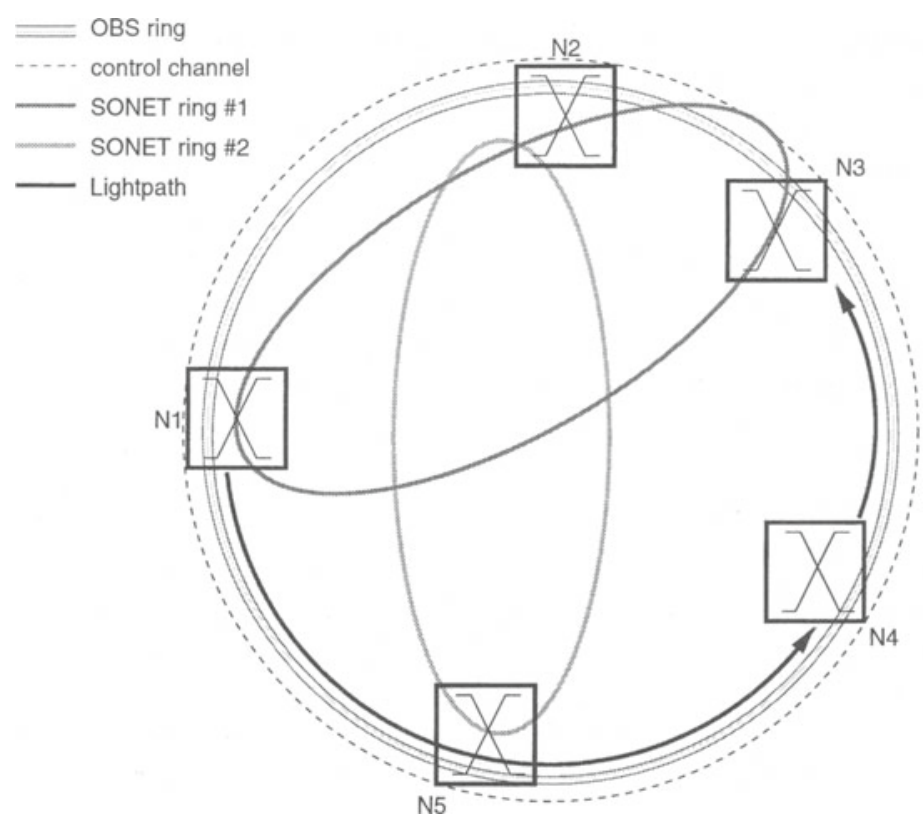

Fig. 1. ORBIS Optical Network Architecture

The ORBIS ring consists of three independent subnetworks: a SONET/SDH ring subnetwork (SONET), a wavelength switched subnetwork (LIGHTPATH), and an optical burst switched subnetwork (OBS). Circuit switching techniques like the SONET/SDH ring and setting up lightpaths are very inefficient to adopt data traffic, but are more suitable for real time traffic. Optical Burst Switching (OBS) has emerged as a more competitive choice for data traffic in the near future $[\mathrm{Xu01}]$.

\section{System Model}

The system is depicted as in the Fig. 1. Each node in the ring will have a minimum of 4 sets of optical transponders. The first is a transceiver fix-tuned to the control channel. The second one is a pair of fast-tunable transmitter and receiver for the BURST subnetwork, which can be tuned to any wavelength independently. The third set is dedicated to the LIGHTPATH and the transceiver can be slowly tuned to a specific wavelength to set up a lightpath between two nodes. The last one is an OADM integrating a set of SONET ADM for the SONET subnetwork. A part of nodes with their OADM tuned to a same wavelength will form a logic SONET/SDH ring. The network structure is depicted in Figure 1.

Each subnetwork will be independently allocated a subset of the total wavelengths, and the transponder for each subnetwork will be given the wavelength 
tuning range according to the allocated wavelengths. At every node in the network, there is an electrical buffer for each subnetwork and incoming traffic is dispatched to a buffer via a QoS-based traffic dispatcher.

There are many interesting problems need to be answered in order to make this architecture a feasible solution, for example, how to allocate the given wavelength resource to the subnetworks. We have developed a set of QoS-based optimal wavelength allocation algorithms based on a game theoretical model [2]. In this paper, we will focus on the control and reconfiguration protocols for the system and each subnetwork.

\section{An Integrated System Control Plane Hierarchy}

The network control plane has two main functions, one is to signal the network elements so that the correct route can be set up and proper resource can be reserved for a traffic requirement. Another is to achieve efficient network management and reconfiguration. In this paper, we consider a distributed control plane to fulfill above functions in our proposed network architecture.

The ring architectures feature simpler synchronization of nodes. This is usually accomplished by a control plan with a slotted control protocol. In the ring with $\mathrm{N}$ nodes, $\mathrm{N}$ control slots, one for each node, make up a control message frame. The control message will continuously circulate around the ring through the dedicated control wavelength.

Since our proposed network architecture consists of multiple subnetworks, a hierarchical control message format is a natural choice, in which, a piece of the message will be in charge of an individual subnetwork. Generally, a host node may write following control information into its slot:

1. Its SONET ring group membership information for the management and reconfiguration of the SONET/SDH subnetwork (details will be given in next section). 2. Lightpath setup, Acknowledgement, and release information. 3. Burst control information including the source and destination address, length, and offset time, etc. 4 . Information about the availability of attached receiver(s). 5 . Other necessary management information related to restoration and security.

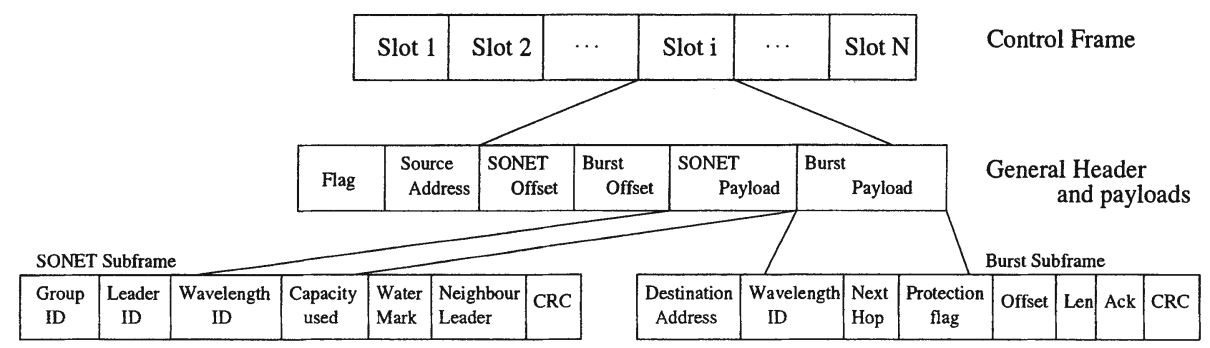

Fig. 2. ORBIS control frame 
The proposed control message structure is depicted in Fig. 2. The general header of a slot provides the offset pointing to part of the payload containing the SONET and Lightpath/Burst sub-frame. The general header also contains the source address, the sub-frame payload length and a flag byte telling the receiving node which types of traffic is coming. The two sub-frames are variable in size. The SONET sub-frame is mainly used for the ring group management and reconfiguration. The Lightpath/Burst sub-frame is mainly used for resource reservation for integrated LIGHTPATH and BURST control.

\section{SONET/WDM Rings}

Since there are multiple rings in the SONET subnetwork, we need to address both ring construction and reconfiguration problem. We assume the BLSR/2 SONET ring structure, i.e. all traffic are routed via shortest paths and half of the capacity are initially reserved for protection/restoration purpose.

The ring construction problem in a WDM platform is usually modeled as a traffic grooming problem, i.e, assembling traffic elements from the given traffic matrix into different SONET rings. A recent survey on this problem can be found in [1]. All of existing studies try to minimize the network resource usage and treat the traffic among all the nodes equally. We argue that nodes with mutual traffic are better to be grouped in one ring due to the management and security concerns and customer requirement. Therefore we propose a novel ring grouping algorithm. It is based on the following ring grouping policies:

1. Those nodes requiring mutual interconnection should be better grouped into the same SONET/SDH ring.

2. Minimize the total number of rings.

3. The transition between different SONET rings should be minimized.

Our grouping problem can be proved to be equivalent the NP-Complet Maximum Clique Problem (MCP) in the graph theory by modeling the traffic matrix as a connected graph. The MCP tries to find the maximum fully connected subgraph (clique) in a simple graph [3]. Each clique found represents a logical SONET ring in the final solution.

To maintain a high utilization of the network resources, a reconfiguration mechanism should be able to change the ring groups by adding and releasing a ring, or migrating traffic between rings, upon the traffic SONET/SDH changes. A novel ring reconfiguration algorithm is given in Fig. 3. We use the backup capacity in the BLSR/2 ring to transfer the existing traffic during the reconfiguration to achieve hitless zero transition loss.

We develop a two-tier control protocol to achieve ring grouping and reconfiguration. The group leader is a special node that is the manager of a logical ring. The control plane for the SONET/WDM rings works in a semi-distributed manner with a two-tier structure. The first tier is for a individual ring under its group leader for the group membership management. The second tier is among 
Input: The initial rings $C=\left(c_{i}\right)$, the watermarks $W_{H}, W_{L}$, the maximum traffic flow $\delta_{i}$ in every ring $c_{i}$

Output: A decision on the ring reconfiguration: adding, removing, or merging.
1. begin
2. Find the minimum ring load $\delta_{\min }=\min \left(\delta_{i}\right)\left(\right.$ ring $\left.c_{\min }\right)$.
3. If $\delta_{\min } \leq W_{L}$ for the required timeout count.
4. If there is another ring $c_{j}$ having the required capacity and allowed membership.
5. Switch the traffic from the work ring to the backup ring.
6. Release the work ring and merge the ring $c_{m i n}$ with ring $c_{j}$.
$7 . \quad$ Release the backup ring.
8. $\quad$ End If
9. $\quad$ End If
10. Find the maximum ring load $\delta_{\max }=\max \left(\delta_{i}\right)\left(\operatorname{ring} c_{\max }\right)$.
11. If $\delta_{m} a x \geq W_{H}$ for the required timeout count.
12. Find the nodes with minimum traffic in $c_{\max }$ untill $\delta_{m} a x \leq W_{H}$.
13. Switch the according traffic to the backup ring and release the working load.
14. Group these nodes into a new ring if the multiplexed traffic $\geq W_{L}$.
15. If not, deliver these traffic to the non-SONET part of ORBIS.
16. Remove the traffic from the backup ring.
17. Update the ring membership.
18. End If
19. end of the algorithm

Fig. 3. Algorithm for ring reconfiguration

all the group leaders. The group leaders communicate with each other in this control tier to make decisions on the ring reconfiguration.

For each type of the two groups, logical ring and the leader group, members can be added or removed dynamically. We propose a multicast-like signaling protocol for the operation of the 2-tier control planes. Each of above group, either the leaders' group or a logical ring group, is considered a multicast group. For each logical ring, the group leader plays the role of the multicast source node. For the leader group, we need to define a hub node to be the source node. We also assume a leaf-initiated joining mechanism.

In this control plane architecture, the two tiers of group membership management are closely cooperative. Initially the leader group is set up with only one member, the hub node. When a new ring is set up, a new group session is setup. Then the group leader is elected which will initiate a ADD PARTY message to join the leader group. When an existing ring needs to be removed, the leader will first initiate a SESSION RELEASE message to release the particular ring group. Then it will initiate a DROP PARTY MESSAGE to the leader group to get itself removed. 


\section{Lightpath and Burst-Switched Subnetwork}

OBS uses a one-way resource reservation mechanism and a pre-sent control packet to set up the light path for a burst transmission session, while setting up a lightpath in the LIGHTPATH subnetwork requires to handle the two-way reservation message. It is obvious that these two tasks can be done by an integrated signaling protocol which will choose the resource reservation method according to the traffic requirement.

we have assumed both tunable transmitter and tunable receivers in each node. However, the tuning time of the receiver is usually much longer. This a fully dynamic case and both receiver and path wavelength assignment must be addressed to set up a lightpath between a pair of transceiver successfully.

The control packet will reserve the wavelength along the path and tune the receiver to the required wavelength. Since the data transmission follows the control packet, the tuning time needs to be included into the offset time. Here we propose a new wavelength reservation protocol, in which 2 control packets are sent out before the data transmission. The first one will not make any wavelength reservation along the path, but directly go to the selected receiver to tune the receiver to the required wavelength if it is free. The second control packet will follow the first one in a later time and will reserve the wavelength along the route. The reservation may be fail either due to the busy receiver (from control packet 1 ), or the occupied wavelength along the route (from control frame 2).

\section{Conclusion}

The focus of this paper is on the control and reconfiguration protocols for the proposed multiple-service WDM ring system and the 3 subnetworks. A set of novel algorithms for ring grouping and reconfiguration and a semi-distributed control protocol for ring membership management are given for the SONET ring subnetwork. For the lightpath and burst subnetworks, a distributed control protocol based on a mixed one-way and two-way reservation protocol is described.

\section{References}

1. Rudra Dutta and George N. Rouskas. Traffic grooming in wdm networks: past and future. IEEE Network 16(6), Nov./Dec. 2002.

2. Yufeng Xin et al. Optimal wavelength allocation in a novel hybrid optical network architecture with a game theoretical approach. In Submitted for publication, 2003.

3. Michael R. Garey and David S. Johnson. Computers and Intractability: A Guide to the Theory of NP-Completeness. W.H. Freeman Co, 1979.

4. N. Ghania, J.-Y. Pan, and X. Cheng. Metropolitan optical networks. Optical Fiber Telecommunications IVB, T. Li (Ed.), 2002. 